\title{
An Approach to Detect Brain Tumor
}

\author{
Prashengit Dhar \\ Department of CSE \\ Port City International University \\ Chittagong, Bangladesh
}

\author{
Md. Burhan Uddin Chowdhury \\ Department of CSE \\ Port City International University \\ Chittagong, Bangladesh
}

\begin{abstract}
Image processing has covered a significant area in medical science. Medical image processing is currently very popular for the effectiveness of image process in medical imaging especially on MR image based task. But it is not so easy. Medical image processing is a challenging task. Wrong decision may cause to great harm to the people. MR images are often used to diagnose and analyze brain tumor. This paper represents a new way for detecting tumor in the brain. This proposed methodology is supported by color information. YCbCr color model is employed for this purpose. Input image is transformed into $\mathrm{YCbCr}$. The segmentation is mainly done by gathering information of Y component. Color based thresholding is performed to segment the image. A morphological action is employed to make the image fine. Then the image is filtered with area. Finally calculated metric value for each object. Highest metric value refers to the tumor object.
\end{abstract}

\section{General Terms}

Brain Tumor; MRI; segmentation; filtering

\section{Keywords}

Metric value; YCbCr; morphological closing

\section{INTRODUCTION}

Among various organ of human body, brain is the most deeply sensitive. Brain tumor is the anomalous progress of cell inside the brain. It is a very risky disease for people. Frequent migraines and headaches are the initial indications of a tumor. Diagnosis of brain tumor is relatively difficult due to diverse size, shape, appearance and location of tumor inside the brain. It is tough to find brain tumor at initial stage. If once tumor is identified earlier then it may be curable. Medical imaging could be beneficial to diagnose the highly noninvasive possibilities. MRI scanning is powerful magnetic fields element to define the radio frequency pulses and to yields the detailed images of soft tissues, organs, bone and various internal structures of body. There are many technologies for medical imaging like-CT scan, X-ray, MRI etc. The MRI process is very effective for detecting brain tumor compared to other. Different image pre-processing methods are used to expand MR image quality.

Tumors are normally spherical shaped. Tgis proposed system makes the best use of this property. Metric value for a round object is 1 or very near to 1 . In a segmented MRI image, basically tumor object is exists as a spherical shaped object. Others object are not so as like as spherical shaped. This is great advantageous in tumor detection for this system. Tumors are fall into two categories according to horizon of tumor. One is primary tumors which are originate in the brain (such as Multiforme, Glioblastoma, Astrocytoma, Meningioma ... etc.). Secondary tumors are extent to the brain though originates from another portion of the body such as Metastatic tumors. Again primary tumors are divided into two classes named as Malignant and Benign. Benign are not cancerous tumors and they rarely grows back. Malignant are cancerous tumor. They grow quicker. Tumor needs to identify at early stage to save patient's life [1].

The paper is presented in an organized way i.e. section 2 demonstrates relevant work of this system, overview of the system is described in section 3 as system overview, the complete detection process is exhibits in section 4 and section 5 is for conclusion with future works.

\section{RELATED WORK}

Beyond the type of tumor, it is mandatory to detect the tumor first. Several methodologies are advised to identify brain tumor. Manoj and Sourabh presented a tumor detection method with the help of histogram, segmentation and thresholding [2]. A method developed by Preetha and Suresh uses fuzzy $\mathrm{C}$ means clustering to segment image [5]. But it consumes time much. The segmentation is identified by SVM. Karuna and Joshi presents an algorithm where segmentation is achieved by Nero Fuzzy Classifier [4]. The system is trained by neural network. A significant number of images were used to train.

A watershed algorithm based segmentation was proposed by Rajesh and Bhalchandra [3]. They also followed morphological operation. A survey on various technique of brain tumor detection is made by Luxit and Sanjeeb [6]. They also discussed about recent image processing methodology which are applicable to medical imaging. . Grosso suggests a supervised learning model along with the pattern recognition manner [9]. They evaluated the decision support system to diagnose brain cancer. The system is supportive to identify the cancer earlier and to classify level of the disease.

Havei and Davvy proposed a different method using deep neural networks for detecting tumor [8]. They investigated different architectures and their performance. Rao and Naganjaneyulu proposed another method which is followed by segmentation, modeling of energy function and optimization of the energy function [7]. They used the information exist in T1 and FLAIR MRI sample images. They used conditional random field for combining information of T1 and FLAIR MRI image. Sridhar classified brain tumor by DCT and probabilistic neural network [10]. Experimental analysis was completed using 20 brain tumor images. The method was relatively faster and provides better recognition rate in contrast with other methods.

\section{SYSTEM OVERVIEW}

The presented paper is about a methodology to detect brain tumor. In this work MRI images are considered to detect brain tumor. The quality of input image is needed to improve.

\subsection{Image Enhance ment}

Image enrichment techniques are broadly utilized in several applications of image processing wherever the subjective quality of picture is vital for human interpretation. Contrast is 
very essential thing about ant subjective analysis of image quality. Contrast is made by the distinction in brightness level from 2 adjacent surfaces. In alternative words, contrast is the dissimilarity in visual properties which distinguishes an object from others and also from the background. Visually contrast is the distinction in brightness and color of the object. Human's sensory system is a lot of sensitive to contrast than the absolute luminance.

Then the image is transformed into $\mathrm{YCbCr}$ color space. The image is then segmented using Y component i.e. a color based thresholding is applied. Later closing is employed on the segmented MR image. Closing is a morphological operation.

\subsection{Morphological ope ration}

Morphological operator typically take a binary image and a structuring part as input image and merge them employing a set operator (union intersection, complement etc.). Objects are processed within the input image supported by characteristics of its form which are determined within the structuring part. Closing is generally opening which executed in reverse. It is outlined merely as a dilation followed by erosion with the help of identical structuring component for each operation. The result of the operator is to preserve the background region that have an identical form of the present structuring part or which will utterly contain the structuring part whereas eliminating all rest of the regions of background pixels. It makes the image fine and better for further processing.
Small objects are removed by filtering the area. Metric value is calculated for each objects. The value for an object closer to 1 or the highest metric value is verified as the tumor object. So brain tumor detection is a collection of various steps.Fig.1 shows the flow chart of explained system.

\section{DETECTION}

Brain tumor detection is a complicated task. The complex brain structure is the reason behind it. This paper proposes a new brain tumor detection approach based on the property of brain MRI and the tumor. In terms of Medical, tumors are spherical. This property leads us finally to detect brain tumor. This study considered MRI image to detect tumor inside the brain. Fig.2, fig.3 and fig.4 demonstrates step by step detection process At first MRI image of brain is taken as input. Fig.2 (a) is an input image sample. To make the image better, some pre-processing steps are employed. Preprocessing is a significant phase in image processing related work. Image is filtered to reduce the noise. An improved and enhanced image results in a good way. Image become more suitable for further various operation. After that image is changed to $\mathrm{YCbCr}$ model. $\mathrm{YCbCr}$ image is shown in fig.2(b). The image is segmented using $\mathrm{Y}$ component. Segmented image is shown $\mathrm{n}$ fig.2(c). A huge amount of images are analy zed and considered 160 as the threshold value.

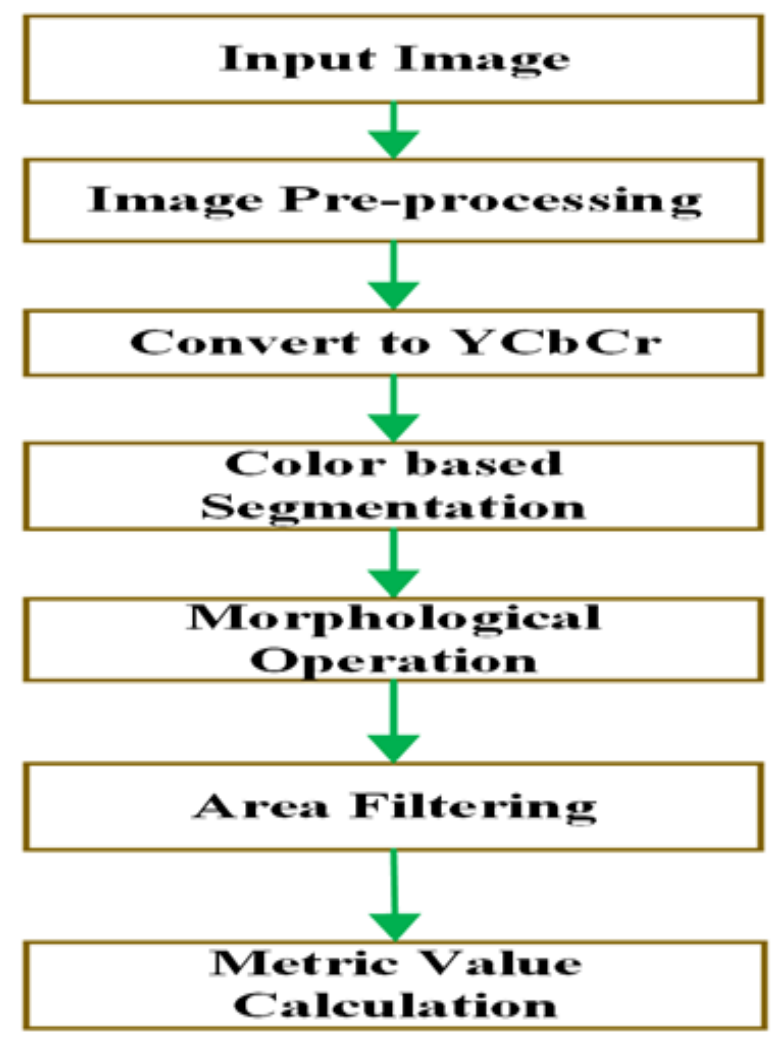

Fig 1: Proposed method 


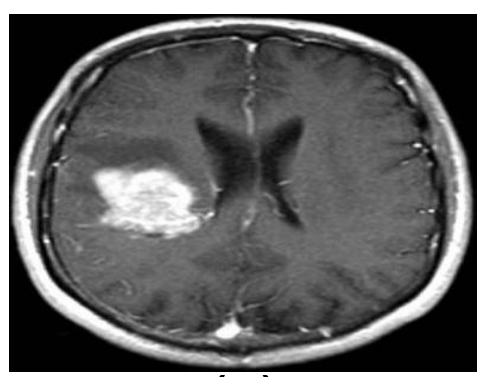

(a)

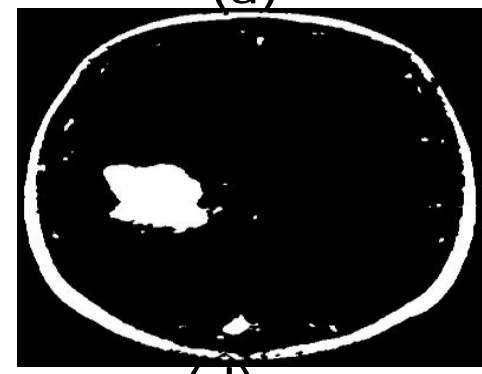

(d)

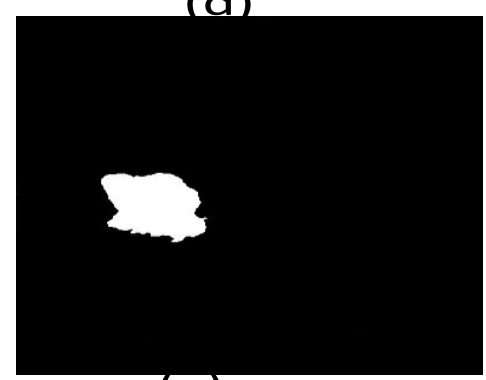

(g)

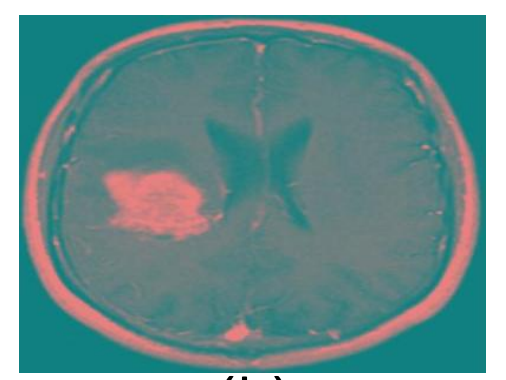

(b)

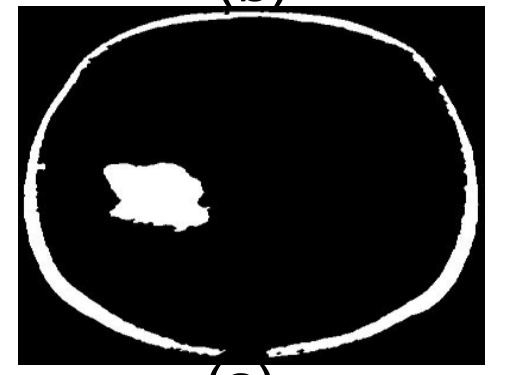

(e)

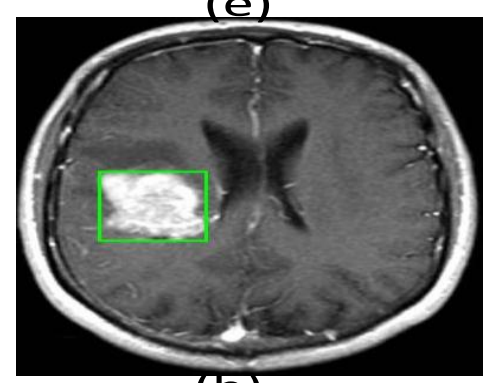

(h)

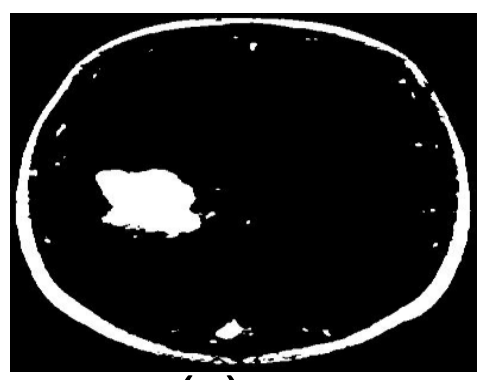

(c)

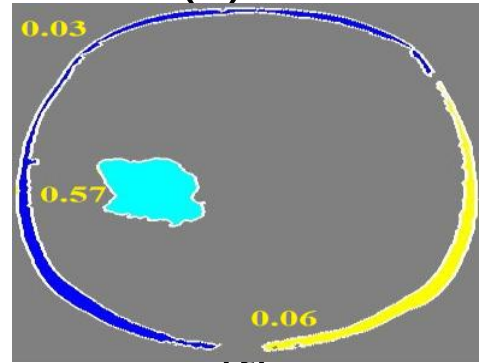

$(\boldsymbol{t})$

Fig 2: Complete detection process (a) input image (b) converted to $\mathrm{YCbCr}$ (c) segmented image (d) after morphological dosing (e) area filtered image (f) metric value calculation (g) detected tumor region (h) located tumor object

Equation 1 is used for segmentation. After this segmentation, a morphological operation on segmented image named as closing is applied. It is used for the recovery of gapes which are smaller than two consecutive pixels. Fig.2 (d) is the output

$\mathrm{I}=\left\{\begin{array}{lr}1 \text { if } Y>160 \\ 0 & \text { else }\end{array}\right.$

image of morphological operation. Morphological closing is a very well and effective operation. Morphological image processing defines a variety of image processing skills that work with the shape (or morphology) of features within an image. Hence morphological operations are usually applied to eliminate imperfections occurred due to segmentation. Some small objects may exists in the image after morphological closing operation. Smaller objects are removed through filtering. Area property is used as filtering criteria. Fig.2 (e) is the filtered image. Then comes an important phase which is the calculation of metric value for each object. A complete round object have a metric value of 1 or very near to 1 . Tumors are like quite spherical object. In a segmented MRI, tumor object is as close as spherical. Finally the object whose metric value is higher is defined as the tumor object. Fig. 2(f) shows metric value of each object. Highest metric value is 0.57 in fig.2 (f). Object with highest metric value i.e. the tumor object is shown in fig. $2(\mathrm{~g})$. Detected tumor is located in fig. 2(h). From fig.2 (f), it can be observed that the metric value for another two existing objects are 0.006 and 0.003 . These values are very low, wherever the metric value of tumor object is 0.57 . Table 1 shows overall statistics of metric value of segmented objects. A threshold value of 0.40 is set by analyzing as minimum metric value for a tumor object.

Fig.3 and fig. 4 also demonstrates step by step tumor detection process. In fig. 3 (f), tumor object has metric value of 0.46 . Other objects metric value are $0.08,009,0.03$ and 0.23 . So the tumor object has the highest metric value. Same thing can also be observed in the example of fig. 4 also. The blue colored object i.e. the tumor object has the maximum metric value. Other objects have lower metric value than tumor object. Table 2 illustrates testing statistics in detecting tumor.

Table 1. Statistics of metric value

\begin{tabular}{|l|l|l|l|}
\hline Fig & $\begin{array}{l}\text { Calculated } \\
\text { metric values }\end{array}$ & $\begin{array}{l}\text { Highest } \\
\text { metric } \\
\text { value }\end{array}$ & $\begin{array}{l}\text { Resultant } \\
\text { metric } \\
\text { value of } \\
\text { tumor } \\
\text { object }\end{array}$ \\
\hline $2(\mathrm{f})$ & $0.03,0.57,0.06$ & 0.57 & 0.57 \\
\hline $3(\mathrm{f})$ & $\begin{array}{l}0.23,0.46,0.08, \\
0.03,0.09\end{array}$ & 0.46 & 0.46 \\
\hline $4(\mathrm{f})$ & $\begin{array}{l}0.05,0.88,0.34, \\
0.36\end{array}$ & 0.88 & 0.88 \\
\hline
\end{tabular}



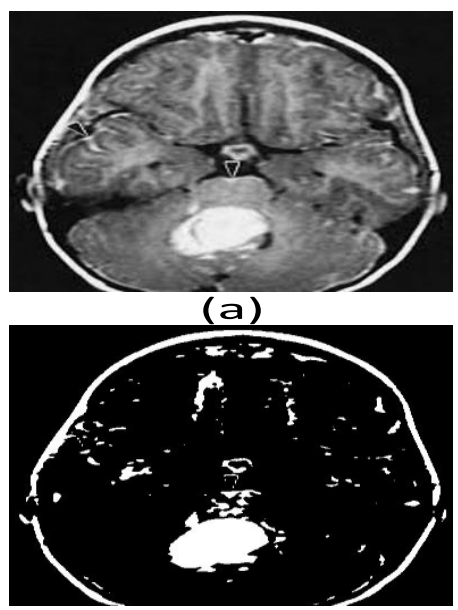

(d)

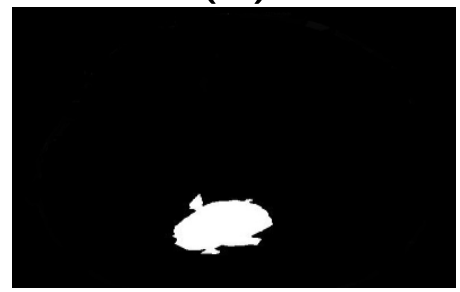

(g)

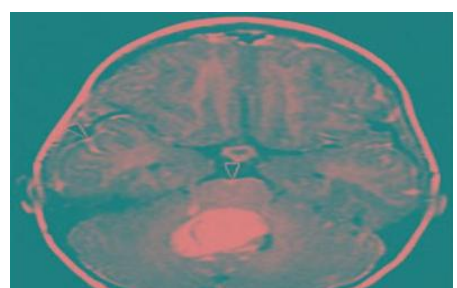

(b)

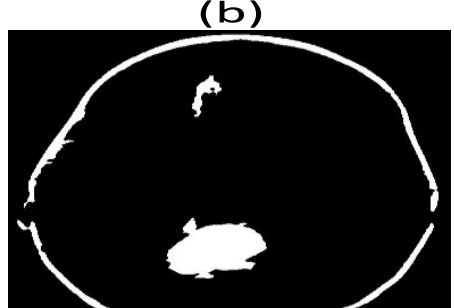

(e)

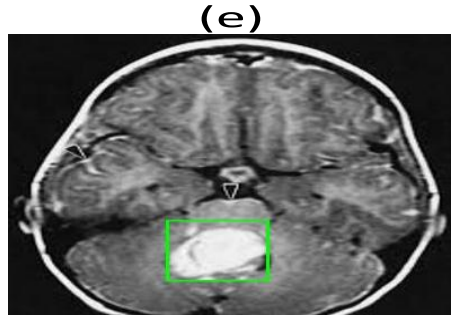

(h)
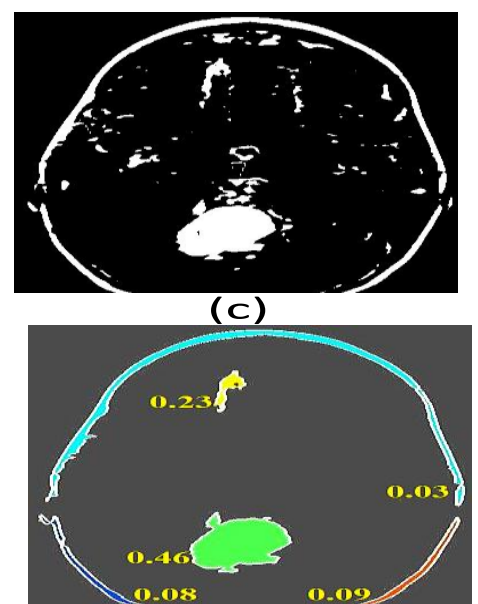

(f)

Fig 3: Complete detection process (a) input image (b) converted to $\mathrm{YCbCr}$ (c) segmented image (d) after morphological dosing (e) area filtered image (f) metric value calculation (g) detected tumor region (h) located tumor object

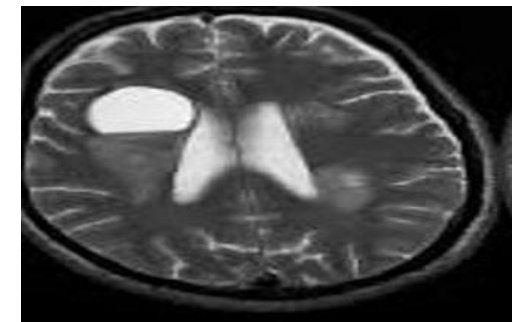

(a)

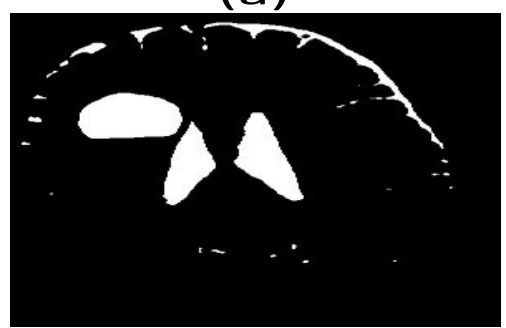

(d)

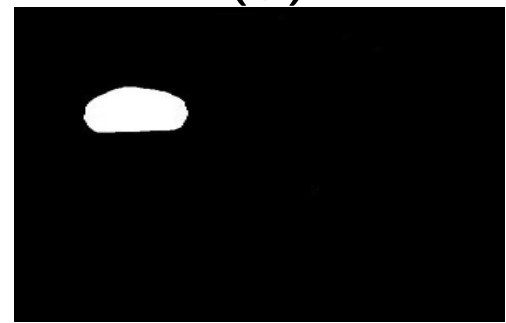

(g)

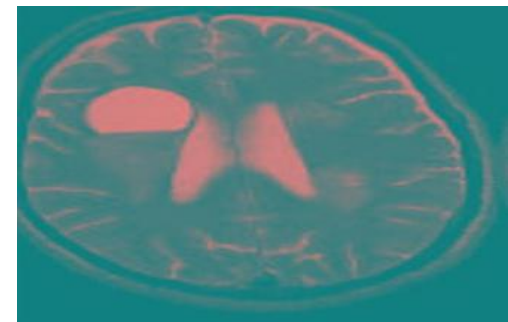

(b)

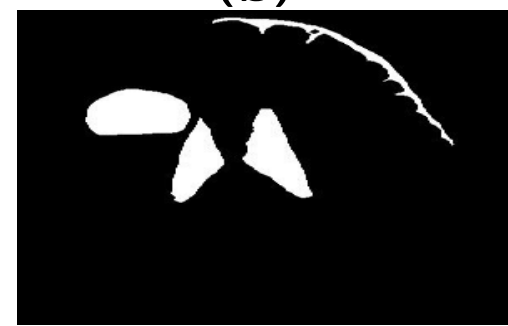

(e)

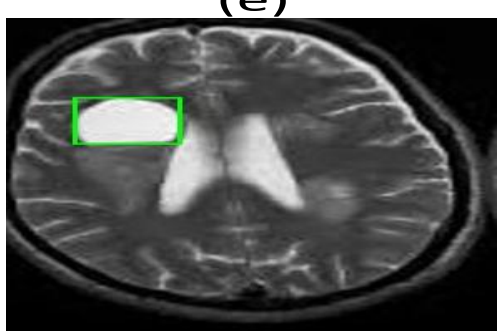

(h)

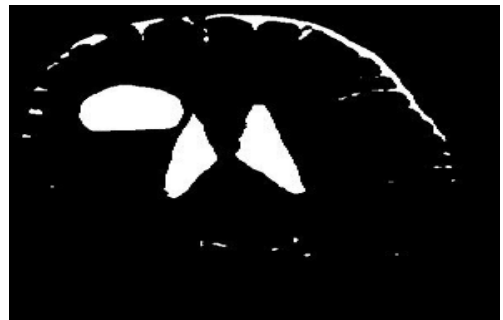

(c)

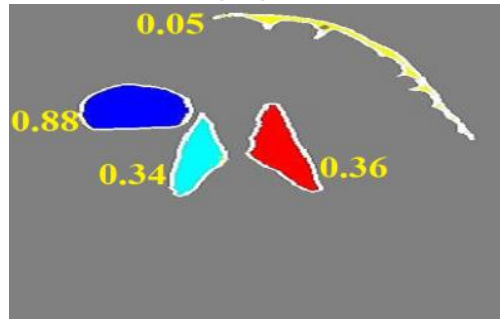

(f)

Fig 4: Complete detection process (a) input image (b) converted to $\mathrm{YCbCr}$ (c) segmented image (d) after morphological dosing (e) area filtered image (f) metric value calculation (g) detected tumor region (h) located tumor object 
Table 2. Testing statistics

\begin{tabular}{|l|l|l|}
\hline Type & $\begin{array}{l}\text { Number of } \\
\text { tested images }\end{array}$ & Detected tumor \\
\hline Images with tumor & 102 & 96 \\
\hline Images without tumor & 33 & 0 \\
\hline
\end{tabular}

\section{CONCLUSION}

A new color based segmentation approach is proposed to detect brain tumor. MRI images are more suitable to detect brain tumor. Image is converted to $\mathrm{YCbCr}$ color model. The segmentation is based on $\mathrm{Y}$ component of $\mathrm{YCbCr}$. It is a new approach introduced in brain tumor detection. A significant number of images have been analyzed to set the threshold value and also to detect tumor. Spherical shape of tumor is a main factor which becomes advantageous in detecting brain tumor. This work is quite different compared to other existing methods. The work is based on color based thresholding process. In future emphasizes will be given on finding out another more efficient method to perform much better.

\section{ACKNOWLEDGEMENT}

Our thanks to Dr. Rashed Mustafa and Md. Zainal Abed in for helping us in this research.

\section{REFERENCES}

[1] S.U. Aswathy, G. Glan Deva Dhas, S.S. Kumar, "A Survey on Detection of Brain Tumor From MRI Brain Images", 2014 international conference on control, Instrumentation, Communication and Computational Technologies(ICCICCT), pp. 871-877, 2014.

[2] Manoj K Kowear and Sourabh Yadev, "Brain tumor detection and segmentation using histogram thresholding", International Journal of engineering and Advanced Technology, April 2012.

[3] Rajesh C. patil, A.S. Bhalchandra, "Brain tumor extraction from MRI images Using MATLab", IJECSCSE, ISSN: 2277-9477, Volume 2, issue 1.

[4] M.Karuna, Ankita Joshi, "Automatic detection and severity analysis of brain tumors using gui in matlab" IJRET: International Journal of Research in Engineering and Technology, ISSN: 2319-1163, Volume: 02 Issue:10,Oct-2013

[5] R. Preetha, G. R. Suresh, "Performance Analysis of Fuzzy C Means Algorithm in Automated Detection of Brain Tumor",IEEE CPS, WCCCT, 2014

[6] Luxit Kapoor, Sanjeev Thakur,"A Survey on Brain Tumor Detection Using Image Processing Techniques", 7th International Conference on Cloud Computing, Data Science \& En gineering, 2017
[7] C.Hemasundara Rao, Dr. P.V. Naganjaneyulu,Dr.K.Satya Prasad, "Brain tumor detection and segmentation using conditional random field", IEEE 7th International Advance Computing Conference, 2017

[8] Mohammad Havaeia,Axel Davy, David Warde-Farley, Antoine Biard, Aaron Courville, Yoshua Ben gio, Chris Pal, "Brain Tumor Segmentation with Deep Neural Networks", arXiv:1505.03540v3 [cs.CV] 20 May 2016

[9] E. Grosso, M.Lopez, C. Salvatore, I. Castiglioni, “ A decision support system for the assisted diagnosis of brain tumors: A feasiblity study for(18)F-FDG PET preclinical studies", 34th Annual international conference of the IEEE EMBS, 2012, pp.6255-6258.

[10] D. Sridhar and Murali Krishna, "Brain tumor classification using discrete cosine transform and probabilistic neural network", IEEE International conference on Signal Processing, Image Processing and Pattern Recognition, 2013, pp. 1-5.

[11] V. SalaiSelvam and S. Shenbagadevi, "Brain Tumor Detection using Scalp EEG with modified Wavelet-ICA and Multi Layer Feed Forward Neural Network", 33rd annual international conference of the IEEE EMBS, IEEE, 2011, pp. 6104-6109.

[12] J. Zhou, K. L. Chan, V. F. H. Chong and S. M. Krishnan, "Extraction of Brain Tumor from MR images using one class support vector machine", 27th annual conference on engineering in medicine and biology, IEEE, pp.64116414.

[13] Yao tienchen, "Brain tumor detection using three dimensional Bayesian level set method with volume rendering", international conference on Wavelet analysis and pattern recognition, IEEE, 2012, pp.158-163.

[14] Mohd Fauzi Othman, Mohd Ariffanan, Mohd Basri, “ Probabilistic Neural network for brain tumor classification", second international conference on intelligent systems, modeling and simulation, IEEE, 2011, 1366-138.

[15] Sahar Ghanavati, Junning Li, Ting Liu, Paul S. Babyn, Wendy Doda and George Lompropoulos, "Automatic brain tumor detection in magnetic resonance images", 9th international conference on Biomedical Imaging, IEEE, 2012, pp. 574-577.

[16] Hongming Li and Yong Fan, "Label propagation with robust initialization for brain tumor segmentation", 9th international symposium on Bio medical imaging, IEEE, 2012, pp.1715-1718. 